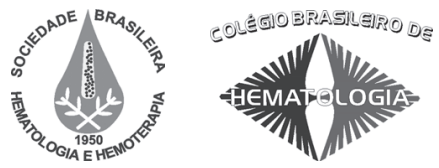

\title{
Mieloma múltiplo: verificação do conhecimento da doença em médicos que atuam na atenção primária à saúde
}

\author{
Multiple myeloma: assessment of knowledge of doctors working in primary healthcare
}

\author{
Roberta O. de Paula e Silval \\ Rosa M. D. de Faria ${ }^{2}$ \\ Maria da Conceição J. W. Côrtes ${ }^{3}$ \\ Nelma C. D. Clementino ${ }^{4}$ \\ José R. de Faria ${ }^{5}$ \\ Thales E. C. Moraes ${ }^{6}$ \\ João L. F. Borges ${ }^{6}$
}

\begin{abstract}
O mieloma múltiplo (MM) apresenta-se clinicamente com sintomas inespecificos que podem não ser identificados associados, dificultando e retardando o diagnóstico. A Atenção Primária à Saúde (APS) é a porta de entrada à rede de assistência à saúde no Brasil, por isso é importante o médico desse serviço reconhecer as características clínicas e laboratoriais do MM para conduzir adequadamente o paciente. Nosso objetivo foi verificar o conhecimento clínico e laboratorial sobre MM em médicos da APS. Foi realizado inquérito epidemiológico utilizando-se teste de múltipla escolha, aplicado em médicos das 137 Unidades Básicas de Saúde de Belo Horizonte (MG), entre outubro e dezembro de 2006. Doenças crônicas e neoplásicas em idosos foram reconhecidas como causas de anemia normocrômica e normocítica por 127 (94,1\%) médicos; lesões osteolíticas ao Raio-X não foram associadas ao MM por 83 (61,5\%); quadro clínico de hipercalcemia não foi clinicamente suspeitado pela maioria, 82 (60,7\%); a eletroforese de proteinas foi incorretamente interpretada por $96(71,1 \%)$ médicos e apenas 49 (36,3\%) pensaram em MM diante de caso clínico característico. O tempo de graduação e de trabalho do médico na APS, assim como a existência de especialização médica, não influenciaram os resultados. Os sinais, sintomas e achados laboratoriais do MM não foram identificados por grande parte da população estudada, sendo um indicador da necessidade de maior interação entre os níveis de atenção secundária e primária, o que proporcionará educação compartilhada e continuada entre os profissionais dos diferentes niveis de atenção à saúde e maior eficiência no cuidado do paciente. Rev. Bras. Hematol. Hemoter. 2008;30(6):437-444.
\end{abstract}

Palavras-chave: Mieloma múltiplo; Atenção Primária à Saúde; diagnóstico.

\section{Introdução}

O MM representa $1 \%$ de todas as neoplasias malignas, sendo a segunda neoplasia hematológica mais comum. ${ }^{1} \mathrm{~A}$ doença caracteriza-se por acometer pacientes idosos, com mediana de idade de 65 anos, no momento do diagnóstico, e raramente acomete pessoas abaixo de 40 anos. ${ }^{2,3} \mathrm{O}$ aumento da incidência dessa neoplasia nos últimos anos relaciona-se a uma série de fatores, entre esses: o maior conhecimento da história natural da doença e sua patogênese, a melhora dos

${ }^{1}$ Professora da Faculdade de Medicina da Universidade Federal de Minas Gerais (UFMG) - Belo Horizonte-MG.

${ }^{2}$ Coordenadora do Curso de Medicina Unifenas-BH. Coordenadora do Atendimento ao Paciente portador de Doença Linfoproliferativa do Serviço de Hematologia do Hospital das Clínicas da UFMG - Belo Horizonte-MG.

${ }^{3}$ Professora Adjunta do DMPS.

${ }^{4}$ Professora Associada convidada do Departamento de Propedêutica Complementar e Médica do Hospital das Clínicas da UFMG e Coordenadora do Ambulatório de Doenças Mieloproliferativas Crônicas do Serviço de Hematologia do HC-UFMG - Belo Horizonte-MG.

${ }_{5}^{5}$ Professor Adjunto do Departamento de Clínica Médica da Faculdade de Medicina da UFMG - Belo Horizonte-MG.

${ }^{6}$ Aluno da Faculdade de Medicina da UFMG - Belo Horizonte-MG.

Faculdade de Medicina da Universidade Federal de Minas Gerais - Belo Horizonte-MG.

Correspondência: Roberta O. de Paula e Silva

Departamento de Propedêtica Complementar, Faculdade de Medicina - UFMG

Av. Prof. Alfredo Balena, 190, sala 6000 - Santa Efigênia

30.130-100 - Belo Horizonte-MG - Brasil

Tel: (+55 31) 3248-9774; (+55 31) 9737-6877; Fax: (+55 31) 3497-4314

Email: betaops@gmail.com 
recursos laboratoriais, o aumento da expectativa de vida mundial e a exposição crônica a agentes poluentes. No Brasil, porém, a incidência de MM é desconhecida, uma vez que a doença não aparece nas estimativas anuais fornecidas pelo Instituto Nacional de Câncer. ${ }^{4} \mathrm{Na}$ cidade de São Paulo, a incidência ajustada para idade do MM entre 1997-1999 era de $3,2 / 100.000$ pessoas do sexo feminino e 4,1/100.000 entre pessoas do sexo masculino. No mesmo período, o MM representou $0,8 \%$ de todos os tumores para homens e mulheres. ${ }^{5}$ Não existe estabelecido um exame de triagem dessa neoplasia para indivíduos assintomáticos, dependendo do conhecimento clínico para se fazer a suspeita diagnóstica.

As manifestações clínicas presentes no MM são consequência direta da infiltração neoplásica medular e da presença da proteína monoclonal no sangue e/ou urina. ${ }^{2,6}$ As principais características clínicas, ao diagnóstico, são: dor óssea, principalmente no dorso e tórax (associada ou não a fraturas patológicas), fraqueza, emagrecimento, infecções recorrentes e alteração da função renal. ${ }^{7}$

A pequena sobrevida apresentada pelos pacientes com diagnóstico tardio é um problema significativo em nosso meio. Estudo realizado na América do Sul por Hungria et al (2005) mostrou que a maioria dos pacientes portadores de MM está em estádio avançado, no momento do diagnóstico: 60 (8\%) estádio I, 91 (12\%) estádio II e 600 (80\%) no estádio III. ${ }^{8}$

Sendo assim, é muito importante o papel do médico da APS no conhecimento da epidemiologia e das principais manifestações clínicas da doença, propiciando a identificação do MM ao avaliar indivíduos que apresentam sinais e sintomas indicativos. O diagnóstico em fases iniciais da doença e a prevenção de complicações impedirão uma pior evolução do paciente, assim como o manejo adequado e o encaminhamento apropriado desses influenciarão na sobrevida e na qualidade de vida dos mesmos. ${ }^{9}$

A proposta desse estudo foi verificar o conhecimento clínico sobre o MM em médicos generalistas ou clínicos de apoio que atuam na rede de APS de Belo Horizonte.

\section{Casuística e Método}

Foi realizado um inquérito epidemiológico, por meio da utilização de um teste de múltipla escolha, tipo melhor resposta. O instrumento foi aplicado a um médico de cada uma das 137 Unidades Básicas de Saúde (UBS) da cidade de Belo Horizonte (MG), com o objetivo de caracterizar o conhecimento e verificar a capacidade de reconhecimento da doença diante de um paciente portador de MM. A escolha desse profissional como sujeito do estudo se justifica por ser este geralmente o responsável pelo primeiro atendimento ao paciente na rede do SUS e, portanto, aquele que primeiro avalia o paciente portador de MM. Cada médico apresentou probabilidade igual e independente de ser incluído na amostra, uma vez que a seleção se deu de forma aleatória, no dia da visita à UBS.
Escolha do instrumento de pesquisa: Optou-se pelo uso de um teste de múltipla escolha, tipo melhor resposta, que consistiu na apresentação de uma situação ou caso clínico com uma pergunta e quatro opções de respostas, uma correta e três distratoras. Como as opções incorretas não são totalmente erradas, solicita-se ao respondedor que escolha a mais correta. Na elaboração do instrumento, cuidou-se de garantir as características fundamentais à medida em ciência: validade (acurácia), reprodutividade e viabilidade.

Construção do instrumento: Foi realizada de maneira seqüencial; para cada questão se determinou um propósito de avaliação (tema) sobre o qual foram elaborados a situação ou caso clínico e as alternativas correta e distratoras. Em seguida, cada alternativa foi avaliada separadamente quanto à lógica e conteúdo. Ao final, o instrumento foi analisado como um todo. As normas técnicas de elaboração de provas de múltipla escolha, tais como tamanho e disposição das alternativas, uso de palavras não indutoras da resposta entre outras, foram seguidas. ${ }^{10,11}$

Com a finalidade de diminuir a possibilidade do respondedor utilizar a eliminação e não o raciocínio aliado ao conhecimento, como forma de obter a resposta correta, as alternativas foram padronizadas, tanto em número quanto em opção de resposta. O instrumento ajustado e liberado para aplicação compunha-se de cinco questões, como mostra o Quadro 1.

De acordo com as questões elaboradas, o instrumento avaliou:

- Questão 1: conduta frente a paciente idoso anêmico.

- Questão 2: diagnóstico diferencial em paciente idoso com lesões osteolíticas ao estudo radiológico convencional.

- Questão 3: capacidade de diagnóstico clínico de hipercalcemia diante de quadro sugestivo.

- Questão 4: capacidade de interpretação da eletroforese de proteínas séricas.

- Questão 5: capacidade de diagnóstico de MM frente a caso clínico característico.

Teste piloto: Após a construção do instrumento foram realizados, no primeiro semestre de 2006, três testes pilotos. O primeiro foi realizado em oito professores de Clínica Médica da Faculdade de Medicina da UFMG, objetivando-se avaliar o conteúdo teórico do mesmo. O segundo e o terceiro testes pilotos foram aplicados em alunos do décimo segundo período da Faculdade de Medicina da UFMG (primeiro teste $n=12$, segundo teste $n=13$ ). Essa população foi escolhi$\mathrm{da}$, uma vez que, sabidamente, boa parte desses alunos inicia a prática médica na APS após a conclusão do curso de graduação, podendo, assim, simular nossa população alvo. Após a última testagem, os ajustes foram concluídos e padronizouse o instrumento para sua aplicação. 
Quadro 1. Instrumento utilizado para verificação do conhecimento sobre mieloma múltiplo em médicos que atuam na Atenção Primária à Saúde de Belo Horizonte

Questionário $n^{\circ}$

Ano da graduação:

Especialidade Médica ( )Sim ( )Não__ual:
Faculdade de origem:

Tempo de trabalho na APS:

1. Diante de um paciente de 70 anos, negro, sexo masculino com anemia normocrômica e normocítica $(\mathrm{Hb}=9 \mathrm{~g} / \mathrm{dl})$, a conduta correta é:

a) Considerar a queda da hemoglobina como um evento fisiológico da senilidade.

b) Investigar a associação da anemia com neoplasias, doença crônica inflamatória e insuficiência renal.

c) Descartar a hipótese de anemia ferropriva, já que esta é hipocrômica e microcítica.

d) Pesquisar deficiência de vitamina B12, que é comum no idoso e possui o padrão morfológico eritrocitário descrito na questão acima.

2. Homem de 54 anos refere dor progressiva na coluna torácica, com irradiação costal há 30 dias. Estudo radiológico revela osteólise e desabamento dos corpos vertebrais de T7 e T8. Esses achados sugerem:
a) Fibrossarcoma
c) Mieloma múltiplo
b) Neoplasia de próstata com metástase óssea
d) Neoplasia de pulmão com metástase óssea

3. Mulher de 72 anos é admitida no Pronto Atendimento com quadro de dores ósseas generalizadas há 4 meses, associadas a letargia, náuseas, vômitos, fraqueza muscular e constipação intestinal há uma semana. Qual dos exames abaixo seria esclarecedor para esse quadro clínico?
a) Dosagem do Cálcio sérico
c) Dosagem do Ácido Úrico sérico
b) Dosagem da Fosfatase Alcalina (fração óssea) sérica
d) Dosagem do Potássio sérico

4. Após observar o perfil eletroforético normal das proteínas séricas (Figura 1), correlacionar a fração das gamaglobulinas das figuras $A, B, C$ e D às situações clínicas descritas abaixo. ATENÇÃO: cada figura ( $A, B, C$ ou $D)$ poderá corresponder a mais de uma situação clínica:

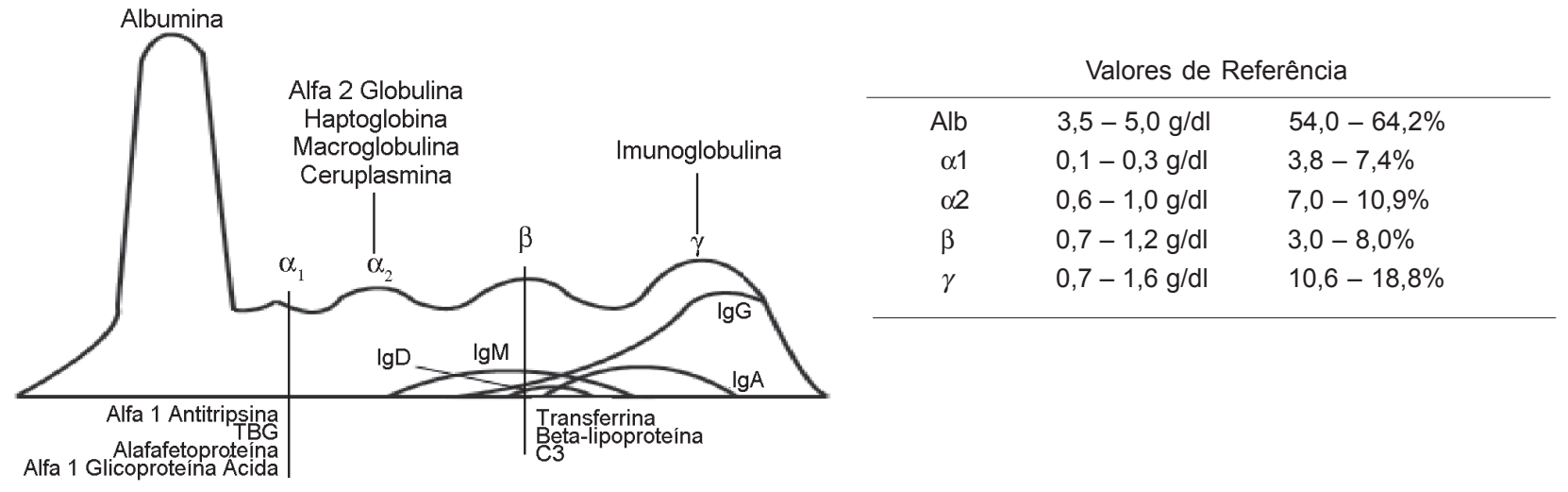

Figura 1. Representação do perfil eletroforético normal das proteínas séricas

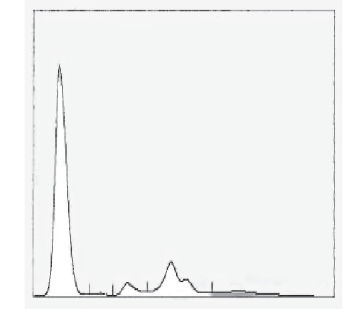

Soroproteinas

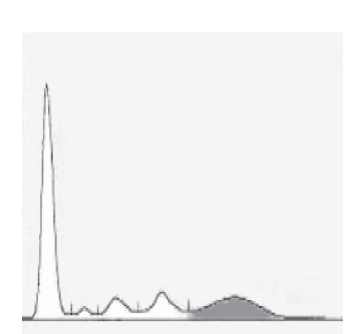

A

\begin{tabular}{l|l|l|}
$\gamma$ & $0,3 \mathrm{~g} / \mathrm{dl}$ \\
\hline
\end{tabular}

C

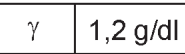

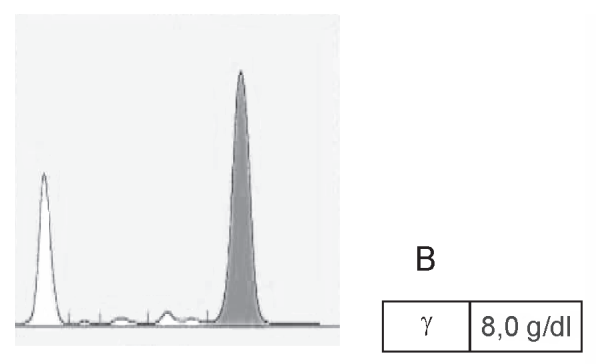

( ) Hipogamaglobulinemia

( ) Mieloma múltiplo

( ) Hipergamaglobulinemia policlonal

( ) Calazar

( ) Hipergamaglobulinemia monoclonal

( ) Neoplasia de próstata com metástase óssea

( ) Neoplasia de pulmão com metástase óssea

( ) Fibrossarcoma

( ) Esquistossomose mansônica forma hepatoesplênica 
5. Homem, 68 anos, comerciante, viúvo, natural e procedente de Belo Horizonte, previamente hígido, há cerca de um ano, após óbito da esposa por câncer de mama, iniciou quadro de astenia, anorexia e perda de peso (10 kg no período), com hipótese diagnóstica de depressão. Na mesma época, sofreu contusão leve na coxa esquerda evoluindo com dor persistente na articulação do quadril ipsilateral, usando antiinflamatório não-hormonal desde então. Foi admitido no Pronto Atendimento com quadro de confusão mental, oligúria, náuseas e vômitos. Tabagista há 20 anos (15 cigarros/dia). Ao exame físico: desidratado ++/4, hipocorado ++/4, emagrecido e com dor a mobilização e limitação funcional na articulação esquerda do quadril.

\begin{tabular}{lcc}
\hline Exames Complementares & Resultados & Valores de Referência \\
\hline Hemoglobina total & $9 \mathrm{~g} / \mathrm{dl}$ & $12,0-17,5 \mathrm{~g} / \mathrm{dl}$ \\
Volume Corpuscular Médio & $86 \mathrm{fl}$ & $80-100 \mathrm{fl}$ \\
Hemoglobina Corpuscular Média & $30 \mathrm{pg}$ & $26-34 \mathrm{pg}$ \\
Cálcio total & $13 \mathrm{mg} / \mathrm{dl}$ & $8,5-10,4 \mathrm{mg} / \mathrm{dl}$ \\
Potássio & $4,2 \mathrm{mEq}$ & $3,7-5,6 \mathrm{mEq}$ \\
Magnésio & $1,4 \mathrm{mEq}$ & $1,6-2,6 \mathrm{mEq}$ \\
Fosfatase Alcalina & $45 \mathrm{Ul}$ & $50-250 \mathrm{Ul}$ \\
Uréia & $120 \mathrm{mg} / \mathrm{dl}$ & $10-40 \mathrm{mg} / \mathrm{dl}$ \\
Creatinina & $2,2 \mathrm{mg} / \mathrm{dl}$ & $0,7-1,2 \mathrm{mg} / \mathrm{dl}$ \\
Albumina & $2,4 \mathrm{~g} / \mathrm{dl}$ & $3,5-5,5 \mathrm{~g} / \mathrm{dl}$ \\
Globulinas & $5 \mathrm{~g} / \mathrm{dl}$ & $1,4-3,2 \mathrm{~g} / \mathrm{dl}$ \\
RX de quadril & Osteólise difusa nos ossos do quadril e & \\
& fratura no colo de fêmur esquerdo & Sem alterações \\
\hline
\end{tabular}

Qual o provável diagnóstico?

a) Fibrossarcoma

b) Neoplasia de próstata com metástase óssea

d) Mieloma múltiplo

e) Neoplasia de pulmão com metástase óssea

Treinamento para aplicação do teste: Dez aplicadores do instrumento foram treinados visando maior precisão dos resultados e eliminação de possível viés de aplicação.

Local de aplicação do instrumento: Ocorreu nas próprias UBS, segundo a disponibilidade de cada médico, no período de outubro a dezembro de 2006.

Análise estatística: Foi feita análise descritiva das variáveis para caracterização do grupo estudado, através da distribuição de frequência, média e desvio padrão. O conhecimento sobre a doença foi definido com base nas questões com maior índice de acertos e aquelas com maior índice de erros. Na análise do porcentual de acerto e erro associado às demais variáveis foi padronizado como erro ou desconhecimento se o médico avaliado optou por não responder a questão, deixando-a em branco. Teste do Qui-quadrado foi utilizado para comparação de frequências das variáveis categóricas entre os grupos codificados. O estudo foi acompanhado, desde seu desenho até sua conclusão, por uma epidemiologista.

Limitações do teste: Apesar de apresentar grandes vantagens, o teste de múltipla escolha, tipo melhor resposta, apresenta algumas limitações. A primeira delas é a limitação de qualquer exame escrito, pois esse não pode medir o desempenho do profissional numa situação real. A segunda, reside na dificuldade de construir um número suficiente de alternativas incorretas, porém plausíveis, na elaboração das alternativas. A terceira limitação se deve ao maior ensejo para palpites pois, como não oferece oportunidade de resposta "livre", exige que o respondedor escolha a resposta correta, dentre as diversas alternativas, possibilitando assim o acerto da questão por sorte, e não por conhecimento. ${ }^{12,13}$

\section{Resultados e Discussão}

A rede de APS em Belo Horizonte é formada por 137 UBS, que se distribuem por nove distritos sanitários da cidade. Foi avaliado um médico de cada UBS (Tabela 1). A amostra final foi de 135 médicos que trabalham na APS da Secretaria Municipal de Saúde de Belo Horizonte, uma vez que em duas UBS não havia atendimento médico no período. ${ }^{14} \mathrm{~A}$ cobertura de toda a rede de APS proporcionou a eliminação do viés de seleção da amostra.

Caracterização da população estudada: O tempo de graduação dos médicos variou de menos de 1 ano a 37 anos, (Média=11,1 anos; DP=9,35). Cinco profissionais optaram por não informar o tempo de graduação e dez dos 130 médicos restantes $(7,7 \%)$ tinham menos de um ano de formados. A distribuição do tempo de graduação dos médicos da APS pode ser vista na Figura 1, e mostra um predomínio de médicos com menor tempo de formado. Atualmente, no Brasil, 
Tabela 1. Divisão regional de Belo Horizonte por Distritos Sanitários com respectivas distribuições de população, médicos, unidades básicas de saúde e testes aplicados por distrito

\begin{tabular}{ccccc}
$\begin{array}{c}\text { Distritos } \\
\text { Sanitários }\end{array}$ & População * $^{*}$ & $\begin{array}{c}\mathrm{N}^{\circ} \text { de } \\
\text { Médicos }^{* *}\end{array}$ & $\begin{array}{c}\mathrm{N}^{\circ} \text { de } \\
\text { UBS }\end{array}$ & $\begin{array}{c}\mathrm{N}^{*} \text { de } \\
\text { testes }\end{array}$ \\
\hline Barreiro & 266.194 & 86 & 20 & 20 \\
Centro-Sul & 266.003 & 58 & 12 & 12 \\
Leste & 249.094 & 65 & 14 & 14 \\
Nordeste & 274.060 & 88 & 21 & 20 \\
Noroeste & 337.351 & 90 & 19 & 19 \\
Norte & 193.764 & 63 & 16 & 16 \\
Oeste & 268.698 & 78 & 15 & 14 \\
Pampulha & 142.602 & 30 & 8 & 8 \\
Venda Nova & 244.566 & 88 & 12 & 12 \\
\hline Total & 2.242 .332 & 646 & 137 & 135
\end{tabular}

Fonte: * IBGE- Instituto Brasileiro de Geografia e Estatística (2006) **SMSA-BH - Secretaria Municipal de Saúde de Belo Horizonte

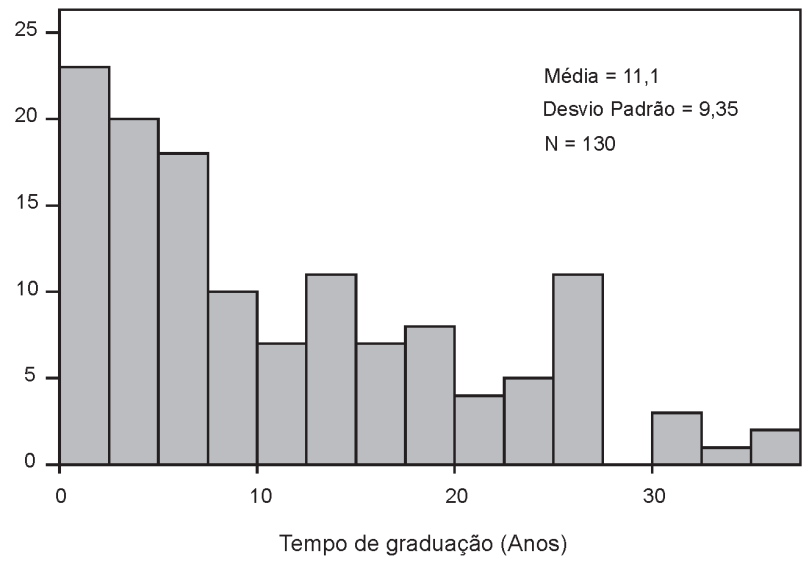

Figura1. Distribuição dos médicos que atuam na Atenção Primária à Saúde de Belo Horizonte de acordo com o tempo de graduação, 2006

existem 20 mil médicos que atuam como generalistas, podendo-se encontrar entre esses desde recém-formados, passando por especialistas das chamadas especialidades básicas, até as especialidades mais particularizadas. ${ }^{15}$

O tempo de trabalho na APS variou de 0,08 anos (um mês) a 30 anos (Média=7,84 anos; $\mathrm{DP}=7,57)$. A distribuição do tempo de trabalho na APS pode ser vista no Gráfico 2 e mostra um predomínio de médicos com menos de cinco anos de trabalho nesse nível de atenção à saúde. Machado (2000) realizou estudo sobre perfil dos médicos do Programa de Saúde da Família (PSF) no Brasil e verificou que 64,32\% tinham entre 5 a 14 anos de formados. Salientamos que o PSF é a principal estratégia do governo brasileiro para viabilização da APS. No mesmo estudo, em relação ao tempo de permanência dos profissionais no PSF, registrou-se que 43,34\% atuavam há menos de um ano como generalistas na APS. ${ }^{16}$

Quanto à presença de especialização, 83 (61,5\%) médicos avaliados possuíam algum tipo de especialização, sendo a mais freqüente Medicina de Família e Comunidade, com 33
$\mathrm{N}^{\mathrm{o}}$

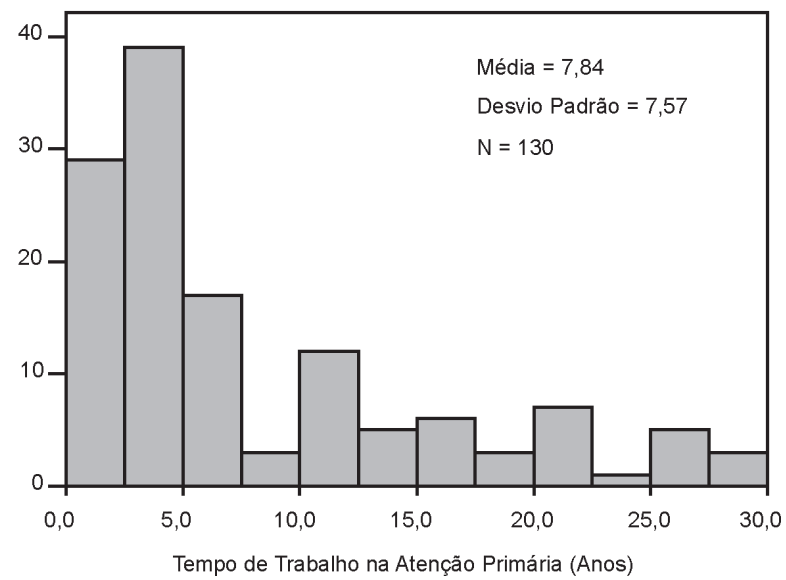

Figura 2. Distribuição dos médicos que atuam na Atenção Primária à Saúde de Belo Horizonte, de acordo com o tempo de trabalho em anos nesse nível de atenção à saúde, 2006

Tabela 2. Especialização dos médicos que atuam na Atenção Primária à Saúde de Belo Horizonte, 2006

\begin{tabular}{lccccc}
\hline \multicolumn{1}{c}{$\begin{array}{c}\text { Tipo de } \\
\text { especialização }\end{array}$} & \multicolumn{2}{c}{ Primeira } & \multicolumn{2}{c}{$\begin{array}{c}\text { Segunda } \\
\text { Especialização }\end{array}$} & \multicolumn{2}{c}{ Especialização } & Total \\
\hline Medicina de Família e & $\mathrm{N}$ & $\%$ & $\mathrm{~N}$ & $\%$ & $\mathrm{~N}$ \\
Comunidade & 23 & 27,7 & 10 & 45,6 & 33 \\
Clínica Médica & 19 & 22,9 & 6 & 27,3 & 25 \\
Pediatria & 13 & 15,3 & 0 & 0 & 13 \\
Ginecologia/ Obstetrícia & 6 & 7,2 & 0 & 0 & 6 \\
Anestesiologia & 4 & 4,8 & 0 & 0 & 4 \\
Cirurgia Geral & 4 & 4,8 & 0 & 0 & 4 \\
Medicina do Trabalhador & 4 & 4,8 & 2 & 9 & 6 \\
Acupuntura e & 2 & 2,4 & 4 & 18,1 & 6 \\
Homeopatia & 8 & 9,6 & 0 & 0 & 8 \\
Outros & 83 & 100 & 22 & 100 & - \\
\hline Total & & & & & \\
\hline
\end{tabular}

médicos, seguida pela Clínica Médica com 25. Vinte e dois médicos possuíam uma segunda especialização (Tabela 2).

\section{Verificação do conhecimento sobre $M M$}

Questão 1: A maioria dos médicos, 127 (94,1\%), concluiu corretamente que, em paciente idoso com anemia normocítica e normocrômica, se faz necessária a avaliação de doenças crônicas e neoplásicas. Esse resultado não foi influenciado pelo tempo de graduação $(\mathrm{p}=0,44)$, tempo de trabalho na APS $(p=0,68)$ ou presença de especialização $(p=0,26)$. Anemia de doença crônica é usualmente definida como a anemia que ocorre em distúrbios infecciosos e inflamatórios crônicos ou em doenças neoplásicas, sendo uma das síndromes clínicas mais comuns e a causa mais frequente de anemia em pacientes com idade superior a 65 anos. Ao diagnóstico, dois terços dos pacientes com MM apresentam anemia, responsável por fraqueza, dispnéia e complicações cardiovasculares, que podem prejudicar a capacidade funcional, a qualidade de vida e a sobrevida desses pacientes. ${ }^{17,18}$ 
Em geral, a gravidade da anemia está relacionada à carga tumoral e pode ser acompanhada de neutropenia e plaquetopenia. ${ }^{2} \mathrm{Na}$ avaliação das alternativas distratoras da questão 1 , a opção predominante foi anemia por deficiência de vitamina B12 (3\%), mostrando que uma minoria abordou a anemia do paciente idoso de forma equivocada nesse caso.

Questão 2: A maior parte dos médicos, 83 (61,5\%), não identificou o MM como principal hipótese diagnóstica, apesar de lesões osteolíticas ao raio-X serem características dessa doença. A principal queixa do paciente portador de $\mathrm{MM}$ é dor óssea. Estudos radiográficos convencionais do esqueleto mostram alterações ósseas, que consistem em lesões líticas, osteoporose e fraturas em $75 \%$ dos casos. Os locais mais frequentes de comprometimento são vértebras, crânio, arcos costais, pelve e porção proximal do úmero e fêmur. Fraturas patológicas são relativamente frequentes, principalmente vertebrais. O comprometimento ósseo influencia consideravelmente a qualidade de vida desses pacientes, levando à dor crônica e, muitas vezes, à incapacidade motora. ${ }^{19}$ Ao avaliar as alternativas distratoras, a neoplasia de próstata foi o diagnóstico diferencial mais marcado isoladamente (23\%). O esqueleto é o terceiro sítio mais frequente de metástase, sendo as neoplasias de próstata, mama, pulmão e rins as que mais comumente levam à metástase óssea. ${ }^{20}$ As metástases desses tumores são osteolíticas e osteoblásticas e é comum ambos os tipos coexistirem no mesmo paciente. No câncer de próstata, aproximadamente $80 \%$ das metástases ósseas são osteoblásticas. No MM, ao contrário, as lesões ósseas são líticas, isto é, ocorre destruição com ausência de neoformação óssea. ${ }^{20,21,22}$ Não houve diferença nos resultados em relação ao tempo de graduação $(\mathrm{p}=0,55)$, tempo de trabalho na APS $(\mathrm{p}=0,84)$ ou presença de especialização $(\mathrm{p}=0,36)$.

Questão 3: A maioria dos médicos avaliados, 82 (60,3\%), não suspeitou clinicamente de hipercalcemia, apesar do caso clínico apresentar manifestações muito sugestivas dessa desordem metabólica. O aumento da reabsorção óssea no MM pode levar à hipercalcemia e em $30 \%$ dos casos o diagnóstico de MM é feito a partir de um quadro clínico característico dessa alteração metabólica. ${ }^{23}$ Os sintomas clínicos comuns a essa desordem são: fadiga, náuseas, vômitos, constipação, fraqueza, letargia, confusão mental e insuficiência renal. Pensar em hipercalcemia e conduzir adequadamente a investigação e tratamento do distúrbio é fundamental para o restabelecimento do paciente. O diagnóstico de hipercalcemia no idoso não é fácil, pois nessa faixa etária várias outras situações clínicas podem justificar tais manifestações. ${ }^{24} \mathrm{~A}$ avaliação das alternativas distratoras mostrou a dificuldade no diagnóstico da hipercalcemia. O diagnóstico diferencial mais marcado, isoladamente, foi hiperpotassemia $(31,1 \%)$. As manifestações clínicas da hiperpotassemia, em geral, limitam- se à esfera neuromuscular e cardíaca. Não houve associação entre $\mathrm{o}$ índice de acerto e a presença de especialização $(\mathrm{p}=0,27)$, o tempo de graduação $(p=0,21)$ e $o$ tempo de trabalho na APS $(\mathrm{p}=0,20)$.

Questão 4: Os tópicos dessa questão avaliaram a interpretação da eletroforese de proteínas séricas em diversas situações clínicas. Como resultado percebeu-se uma dificuldade generalizada na interpretação deste exame laboratorial e na sua correlação com situações da prática médica. Em relação ao MM, a soma das porcentagens de erro e não resposta foi de $71,1 \%$ (96), evidenciando dificuldade na interpretação do perfil eletroforético, não só no $\mathrm{MM}$, mas em outras situações clínicas. A eletroforese de proteínas séricas é um exame laboratorial simples, de fácil realização e passível de utilização em nível de APS na rede de Apoio Diagnóstico da SMSA-BH. ${ }^{14}$ Esse método permite separar as proteínas do plasma humano em frações e sua interpretação traz informações úteis ao médico, o que o torna importante ferramenta para investigação e diagnóstico de diversas doenças, principalmente as gamopatias monoclonais. $O$ resultado expressa quantitativamente as frações de proteínas séricas albumina, alfa-1 globulinas, alfa-2 globulinas, betaglobulinas e gamaglobulinas, em valores absolutos e relativos. Necessariamente, o resultado da eletroforese precisa conter o registro gráfico da corrida eletroforética, para possibilitar a caracterização da curva e/ou pico que retrata a mobilidade da proteína ou do grupo de proteínas. ${ }^{25} \mathrm{~A}$ eletroforese de proteínas séricas e/ ou urinárias é fundamental no diagnóstico e seguimento clínico dos pacientes com MM. ${ }^{26}$ Pico monoclonal na fração das gamaglobulinas pode ser detectado pela eletroforese de proteínas séricas em até $80 \%$ dos pacientes portadores de MM. ${ }^{23}$ A confirmação da proteína monoclonal é essencial para diferenciar gamopatias monoclonais (pico com base estreita) das gamopatias policlonais (pico com base larga), uma vez que as primeiras são entidades neoplásicas ou potencialmente neoplásicas, enquanto as últimas resultam de processos reacionais. ${ }^{27}$ Não houve diferença no índice de acerto dessa questão em relação à presença de especialização $(\mathrm{p}=0,55)$, tempo de graduação $(\mathrm{p}=0,88)$ e tempo de trabalho naAPS $(\mathrm{p}=0,74)$.

Questão 5: Apenas 49 (36,3\%) médicos formularam hipótese de MM frente ao quadro clínico característico. Os sinais e sintomas mais freqüentes no paciente portador de MM, ao diagnóstico, são: dor óssea difusa, fraqueza, fraturas patológicas, infecções recorrentes e alterações da função renal, sendo a tríade clássica formada por anemia, fraqueza e dores ósseas. ${ }^{28}$ Alteração renal está presente em $20 \%$ a $60 \%$ dos casos de MM ao diagnóstico e pode ser intensificada pelo uso de antiinflamatórios não-esteróides, hipercalcemia e desidratação. ${ }^{29}$ Estudos radiográficos convencionais mostram lesões líticas em $67 \%$ dos casos, e osteoporose, fratura patológica e compressão medular em 20\%.3,30 
O diagnóstico diferencial mais marcado isoladamente foi neoplasia de próstata $(30,4 \%)$. Apenas $2 \%$ dos pacientes com tumores de próstata apresentam metástases para o osso e estas, na maioria das vezes, são osteoblásticas e tardias. ${ }^{31}$ Em contraste, no MM, o acometimento ósseo ocorre em cerca de $60 \%$ dos pacientes, a lesão é caracteristicamente osteolítica, a dor óssea faz parte da sintomatologia inicial do paciente e comumente está acompanhada de fraqueza e anemia. Não houve associação entre o índice de acerto e a presença de especialização $(\mathrm{p}=0,187)$, o tempo de graduação $(\mathrm{p}=0,652)$ e $\mathrm{o}$ tempo de trabalho na APS $(\mathrm{p}=0,192)$.

\section{Conclusões}

Grande parte da população médica que atua na APS da cidade de Belo Horizonte não identificou aspectos clínicos e laboratoriais característicos do MM. O pouco conhecimento sobre a doença identificado é homogêneo em toda essa rede de APS, não sofrendo influência do tempo de graduação, tempo de trabalho na APS ou presença de especialização médica. Esse resultado é um importante indicador da necessidade de maior interação entre os níveis de atenção secundária e primária, o que proporcionará educação compartilhada e permanente entre os profissionais dos diferentes níveis de atenção à saúde e, conseqüentemente, maior eficiência no cuidado do paciente.

Propomos um fluxograma para abordagem do paciente com sintomatologia sugestiva de MM, passível de ser utilizado por médicos que atuam na APS, tendo como principais

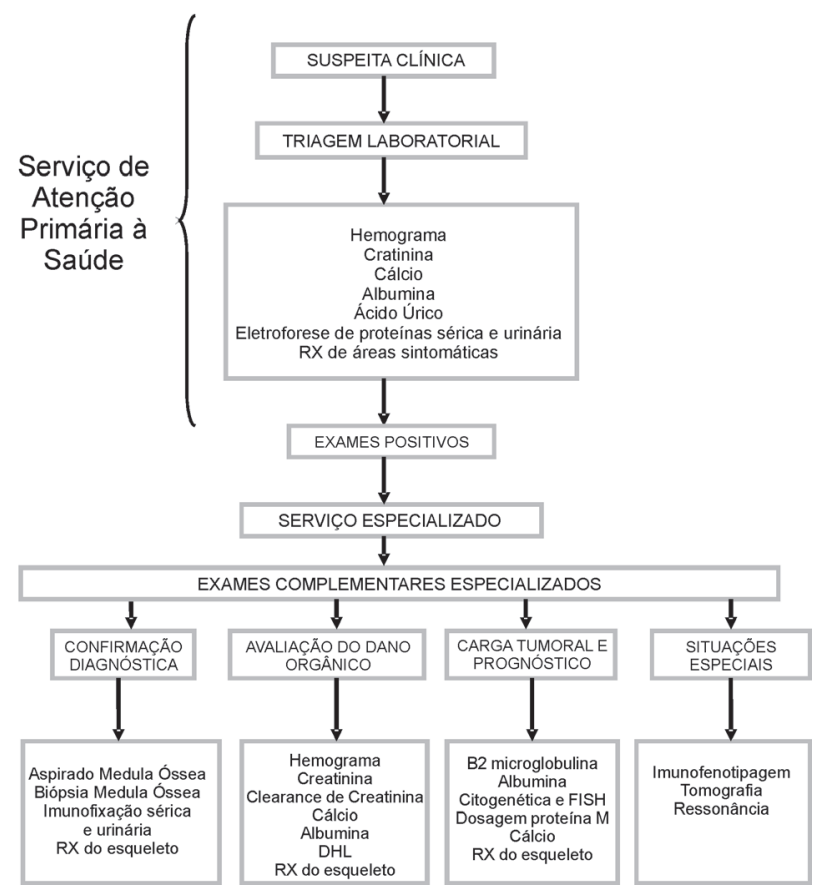

Figura 3. Fluxograma para abordagem diagnóstica do paciente com suspeita clínica de MM (Faria \& Silva, 2007) objetivos uma maior eficiência no compartilhamento do cuidado e a interação educativa entre os dois níveis de atenção à saúde, mais comumente utilizado pelo portador de MM no Brasil, o primário e o secundário (Figura 3).

\section{Abstract}

Multiple myeloma (MM) presents clinically with unspecific symptoms with an association that may not be identified, thus delaying the difficult diagnosis. Primary healthcare (PHC) is the doorway to the health system in Brazil; hence, doctors in this service should be able to recognize clinical and laboratorial characteristics of $M M$, in order to manage the patient properly. Our objective was to verify clinical and laboratorial knowledge on MM of doctors working in PHC. An epidemiological survey was performed using a multiplechoice test completed by doctors of the 137 PHC units in Belo Horizonte $(M G)$, between October and December 2006. Chronic and neoplastic diseases were recognized as causes of normochromic and normocytic anemia by 127 (94.1\%) doctors; osteolytic lesions in the X-ray were not associated to MM by 83 (61.5\%); clinical manifestations of hypercalcemia were not a clinical suspicion for the majority (82 - 60.7\%); protein electrophoresis was incorrectly interpreted by $96(71.1 \%)$ doctors and only 49 (36.3\%) considered MM when presented with a characteristic clinical case. Neither time since graduation, nor time working in PHCs, nor medical specialty influenced the results. A large proportion of the studied population failed to identify the signs, symptoms and laboratorial findings of $M M$, which indicates the need for a greater interaction between secondary and primary healthcare professionals, which will, in turn, promote shared and continuous education among professionals at the different levels of healthcare and a higher efficiency in patient care. Rev. Bras. Hematol. Hemoter. 2008;30(6):437-444.

Key words: Multiple myeloma; Primary Health Care; diagnosis.

\section{Referências Bibliográficas}

1. Hussein MA, Juturi JV, Lieberman I. Multiple myeloma: present and future. Curr Opin Oncol. 2002;14(1):31-5.

2. Mangan P. Recognizing multiple myeloma. Nurse Pract. 2005; 30(3):14-27

3. Kyle RA et al. Review of 1027 patients with newly diagnosed multiple myeloma. Mayo Clin Proc. 2003;78(1):21-33.

4. Brasil. Ministério da Saúde. Instituto Nacional do Câncer. Estimativa 2006: Incidência de Câncer no Brasil. Disponível em: http:// www.inca.gov.br/estimativa/2006. Acesso em: 21 jun. 2007.

5. Mirra AP, Latorre MRDO, Veneziano DB. Aspectos epidemiológicos do câncer no município de São Paulo. Fatores de risco, 2003. São Paulo: Registro de Câncer de São Paulo, 2003. Disponível em: http://hygeia.fsp.usp.br/rcsp. Acesso em: 21 jun. 2007.

6. Durie BGM. International Myeloma Foundation: Revisão resumida do mieloma e das opções de tratamento. p.1-28, 2005. Disponível em: http://www.mielomabrasil.org/publicacoes.php. Acesso em: 21 jun. 2007

7. Sousa FACV et al. Mieloma múltiplo: uma breve revisão. Revista Brasileira de Patologia Oral. 2004;3(4):176-9.

8. Hungria VT et al. South American Multiple Myeloma Study: Epidemiological and clinical characteristics of 751 patients. Haematologica. 2005;90 (s1):abstract 521. 
9. Dvorak C. Common complaints, difficult diagnosis: multiple myeloma. J Am Acad Nurse Pract. 2006;18(5):190-4.

10. Case SM, Swanson DB. Constructing Written Test Questions for the basic and clinical sciences. Filadélfia: Editora Copyright. 180 p, 1998.

11. Morrison S, Free KW. Writing multiple-choice test items that promote and measure critical thinking. J Nurs Educ. 2001;40 (1):17-24

12. Anderson J. Multiple-choice questions revised. Med Teach. 2004; 26(2):110-3.

13. Roediger HL 3rd, Marsh EJ. The positive and negative consequences of multiple-choice testing. J Exp Psychol Learn Mem Cogn. 2005; 31(5):1155-9.

14. Secretaria Municipal de Saúde de Belo Horizonte (SMS-BH). Gerência em Epidemiologia e Informação. Disponível em: <http:// portal1.pbh.gov.br/pbh/pg Acesso em: 21 jun. 2007

15. Aleixo JLM. A Atenção Primária à Saúde e o Programa de Saúde daFamília: Perspectivas de desenvolvimento no início do terceiromilênio. Revista Mineira de Saúde Pública. 2002;1:19-25.

16. Machado MH. Perfil dos Médicos e Enfermeiros de Saúde da Família no Brasil. Disponível em: <http://www.ensp.fiocruz.br/ psf_perfil/index.html> Acesso em: 21 jun. 2007

17. Birgegård G, Gascón P, Ludwig H. Evaluation of anaemia in patients with multiple myeloma and lymphoma: findings of the European Cancer Anaemia Survey. Eur J Haematol. 2006;77(5):378-86.

18. Cançado RD. Mieloma múltiplo e anemia. Rev Bras Hematol Hemoter. 2007;29(1):67-76.

19. Hungria VTM. Doença óssea em mieloma múltiplo. Rev Bras Hematol Hemoter. 2007;29(1):60-6.

20. Farias MLF. A hipercalcemia nas malignidades: aspectos clínicos, diagnósticos e terapêuticos. Arq Bras Endocrinol Metab. 2005;49 (5):816-24.

21. Poulos AR, et al. Pain, mood disturbance, and quality of life in patients with multiple myeloma. Oncol Nurs Forum. 2001; 28(7):1163-71.
22. Huang E, et al. Recurrence of prostate adenocarcinoma presenting with multiple myeloma simulating skeletal metastases of prostate adenocarcinoma. Urology. 2002;60(6):1111.

23. Barose G, et al. Management of multiple myeloma and relateddisorders: guidelines from the Italian Society of Hematology (SIE), Italian Society of Experimental Hematology (SIES) and Italian Group for Bone Marrow Transplantation (GITMO). Haematologica. 2004;89(6):717-41.

24. Zaidi AA, Vesole DH. Multiple myeloma: an old disease with new hope for the future. CA Cancer J Clin. 2001;51(5):273-85.

25. Bottini PV. Testes laboratoriais para avaliação do componente monoclonal. Rev. Bras Hematol Hemoter. 2007;29(1):23-6.

26. Rajkumar SV, Kyle RA. Multiple myeloma: diagnosis and treatment. Mayo Clin Proc. 2005;80(10):1371-82.

27. Kyle RA. Sequence of testing for monoclonal gammopathies. Arch Pathol Lab Med. 1999;123(2):114-8.

28. Dispenzieri A, Kyle RA. Multiple myeloma: clinical features and indications for therapy. Pract Res Clin Haematol. 2005;18(4): 553-68.

29. Maiolino A, Magalhães RJP. Mieloma múltiplo e insuficiência renal. Rev. Bras. Hematol. Hemoter. 2007;29(1):86-91.

30. Faria RMD, Silva ROP. Gamopatias monoclonais - critérios diagnósticos e diagnósticos diferenciais. Rev. Bras. Hematol. Hemoter. 2007;29(1):17-22.

31. Roodman GD. Pathogenesis of myeloma bone disease. Blood Cells Mol Dis. 2004;32(2):290-2.

Avaliação: Editor e dois revisores externos

Conflito de interesse: não declarado

Recebido: 14/05/2008

Aceito após modificações: 30/05/2008 